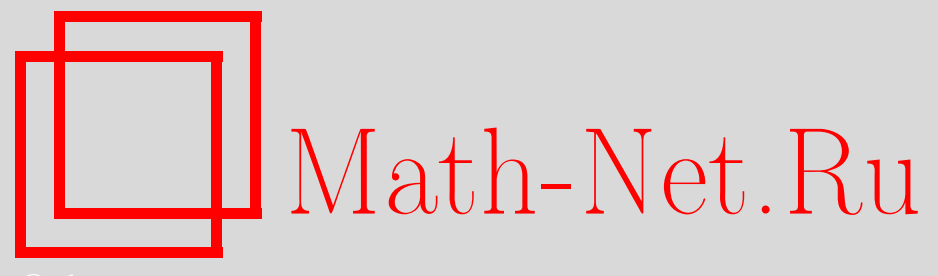

Е. В. Орлова, О сложности реализации конечных языков формулами, Дискрет. матем., 2000, том 12, выпуск $1,145-157$

DOI: https://doi.org/10.4213/dm319

Использование Общероссийского математического портала Math-Net.Ru подразумевает, что вы прочитали и согласны с пользовательским соглашением http://www.mathnet.ru/rus/agreement

Параметры загрузки:

IP : 3.81 .55 .215

26 апреля 2023 г., $17: 54: 06$

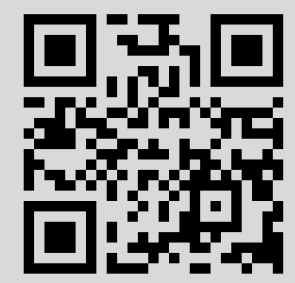




\title{
О сложности реализации конечных языков формулами
}

(C) 2000 г. Е.В. Орлова

\begin{abstract}
Рассматривается реализация конечных языков регулярных выражений в конечных алфавитах формулами, имеющими минимальную сложность. Исследованы некоторые классы языков, для которых получены наилучшие по порядку оценки. Основное внимание уделено реализации языков, состоящих из слов одинаковой длины.
\end{abstract}

\section{1. Основные определения}

Словом в алфавите называется конечная последовательность букв этого алфавита. Языком в алфавите называется множество слов в этом алфавите.

Язык, состоящий из слов одинаковой длины, будем называть однородным. Однородный язык с длиной слова равной $n$, будем называть $n$-однородным. Без ограничения общности, под алфавитом будем понимать алфавит $E_{k}=\{0,1, \ldots, k-1\}$, $k \geqslant 2$.

Языки регулярных выражений $[1,2]$ строятся следующим образом. Вводятся операции $U, \times$ и $*$, имеющие следующую интерпретацию.

Пусть $M_{1}$ и $M_{2}$ - произвольные языки. Через $M_{1} \cup M_{2}$ обозначают объединение языков $M_{1}$ и $M_{2}$, а через $M_{1} \times M_{2}$ - множество всех слов, каждое из которых является конкатенацией слова из $M_{1}$ и слова из $M_{2}$. Через $* M_{1}$ обозначается

$$
\bigcup_{i=1}^{\infty} M_{1}^{i}
$$

где

$$
M_{1}^{1}=M_{1}, \quad M_{1}^{2}=M_{1} \times M_{1}, \quad M_{1}^{n}=M_{1}^{n-1} \times M_{1}, \quad n>2 .
$$

Язык регулярных выражений определяется индуктивно следующим образом.

1. Множество, состоящее из буквы алфавита, является языком регулярных выражений.

2. Если $M_{1}$ и $M_{2}$ - языки регулярных выражений, то $M_{1} \cup M_{2}, M_{1} \times M_{2}$ и $* M_{1}$ также являются языками регулярных выражений.

3. Других языков регулярных выражений нет. 
Таким образом, каждый язык регулярных выражений можно задать формулой над базисом $\{\cup, \times, *\}$. Результатом применения операции * является язык регулярных выражений, содержащий слова разной длины, причем существуют слова, длина которых превосходит любое наперед заданное число. Таким образом, при построении языков со словами ограниченной длины операция * неприменима. Отметим, что операция $\cup$ не изменяет длины слов, а операция $\times$ увеличивает длины слов.

Всюду в дальнейшем под формулой будем понимать формулу над базисом $\{\cup, \times\}$. Под сложностью формулы будем понимать число операций $\cup$ и $\times$. Через $L_{\Phi}(\mathscr{L})$ обозначим минимум сложностей формул, задающих язык $\mathscr{L}$, а через $L_{\Phi}(M)-$ максимум $L_{\Phi}(\mathscr{L})$ по всем языкам $\mathscr{L}$ из множества $M$. Пусть $L_{\Phi}(k, n)=L_{\Phi}(M)$, где $M$ - множество языков в алфавите $E_{k}$ с максимальной длиной слова, равной $n$, и пусть $L_{\Phi}^{0}(k, n)=L_{\Phi}\left(M^{0}\right)$, где $M^{0}$ - множество $n$-однородных языков в алфавите $E_{k}$.

Двухполюсная последовательно-параллельная сеть, все дуги которой ориентированы естественным образом (от входа к выходу), называется $\pi$-сетью; $\pi$-сеть, дугам которой приписаны буквы алфавита $E_{k}$, будем называть $(\pi, k)$-схемой (в случае $k=2$ будем называть ее $\pi$-схемой).

Формуле в алфавите $E_{k}$ поставим в соответствие $(\pi, k)$-схему. При этом операции $\cup(\times)$ соответствует параллельное (последовательное) соединение подсхем. Формуле $\alpha, \alpha \in E_{k}$, ставим в соответствие $(\pi, k)$-схему, состоящую из инцидентной ее входу и выходу дуги, которой приписан символ $\alpha$.

Таким образом, формуле соответствует единственная $(\pi, k)$-схема.

Пусть формула $\Phi$ задает язык $\mathscr{L}$ в алфавите $E_{k}$. Скажем, что $(\pi, k)$-схема, соответствующая формуле $\Phi$, реализует язык $\mathscr{L}$.

Под сложностью $(\pi, k)$-схемы будем понимать число ее дуг. Через $L_{\pi}(\mathscr{L})$ обозначим минимум сложностей $(\pi, k)$-схем, реализующих язык $\mathscr{L}$ в алфавите $E_{k}$, а через $L_{\pi}(M)$ - максимум $L_{\pi}(\mathscr{L})$ по всем языкам $\mathscr{L}$ из множества $M$. Пусть $L_{\pi}(k, n)=L_{\pi}(M)$, где $M-$ множество языков в алфавите $E_{k}$ с максимальной длиной слова, равной $n$, и пусть $L_{\pi}^{0}(k, n)=L_{\pi}\left(M^{0}\right)$, где $M^{0}-$ множество $n$-однородных языков в алфавите $E_{k}$. При $k=2$ индекс $k$ будем опускать.

Известно, что для любого конечного языка $\mathscr{L}$ имеет место равенство

$$
L_{\pi}(\mathscr{L})=L_{\Phi}(\mathscr{L})+1
$$

Через $N_{f}$ обозначаем множество наборов, на которых булева функция $f$ равна 1 . Таким образом, булевой функции $f$ от $n$ переменных соответствует являющийся $n$-однородным язык $N_{f}$ в алфавите $E_{2}$. Ввиду этого, не будем различать булеву функцию $f$ и соответствующий ей (однородный) язык $N_{f}$. Следовательно, вопрос о реализации однородных языков в алфавите $E_{2}$ регулярными выражениями сводится к задаче реализации булевых функций (однородными) $\pi$-схемами. Это дает возможность использовать достаточно хорошо разработанную теорию оптимального синтеза булевых функций.

Пусть $D_{n}-$ множество всех дизъюнкций $x_{1}^{\sigma_{1}} \vee x_{2}^{\sigma_{2}} \vee \ldots \vee x_{n}^{\sigma_{n}}$, пусть $F_{k, n, r}$ множество всех $n$-однородных языков в алфавите $E_{k}$ мощности $r$ и пусть $D_{k, n}=$ $F_{k, n, k^{n}-1}$.

В работе получены оценки функционалов $L_{\Phi}^{0}(k, n), L_{\Phi}(k, n), L_{\Phi}\left(D_{n}\right), L_{\Phi}\left(D_{k, n}\right)$ и $L_{\Phi}\left(F_{k, n, r}\right)$.

Для простоты изложения приведены доказательства утверждений о сложности реализации языков в алфавите $E_{2}$. 


\section{2. Асимптотические оценки функционала $L_{\pi}^{0}(n)$}

Основными результатами этого параграфа являются доказательство соотношения $L_{\pi}^{0}(n) \sim \sigma 2^{n}$ и получение оценок константы $\sigma$.

Теорема 1. $\Pi p u n \rightarrow \infty$

$$
L_{\pi}^{0}(n) \sim \sigma 2^{n}
$$

где $\sigma$ - некоторая константа.

Доказательство. Пусть $N(l)$ - число неизоморфных $\pi$-схем, имеющих $l$ дуг. Из оценки числа $\pi$-сетей $\left[7\right.$, стр. 173] нетрудно получить оценку $N(l) \leqslant 2^{5 l}$, из которой, применяя обычные мощностные рассуждения, получаем соотношение

$$
L_{\pi}^{0}(n) \gtrsim \frac{1}{5} 2^{n}
$$

Нетрудно проверить, что для любого натурального $n$ имеет место соотношение

$$
L_{\pi}^{0}(n+1) \leqslant 2 L_{\pi}^{0}(n)+2 .
$$

Представим $L_{\pi}^{0}(n)$ в виде $\sigma(n) 2^{n}$. Из (3) получаем, что

$$
\sigma(n+1) \leqslant \sigma(n)+\frac{2}{2^{n+1}}=\sigma(n)+2\left(\frac{1}{2^{n}}-\frac{1}{2^{n+1}}\right)
$$

или

$$
\sigma(n+1)+\frac{2}{2^{n+1}} \leqslant \sigma(n)+\frac{2}{2^{n}}
$$

Пусть

$$
\sigma^{\prime}(n)=\sigma(n)+\frac{2}{2^{n}} .
$$

Из (4) следует, что последовательность $\left\{\sigma^{\prime}(n)\right\}$ монотонно убывает. Последовательность $\{\sigma(n)\}$ ограничена снизу константой $1 / 5$. Следовательно, последовательность $\left\{\sigma^{\prime}(n)\right\}$ также ограничена снизу и по теореме Вейерштрасса имеет предел. Таким образом, последовательность $\sigma(1), \sigma(2), \ldots, \sigma(n), \ldots$ имеет тот же самый предел, который мы обозначим через $\sigma$.

Теорема 1 доказана.

Произвольную булеву функцию от $n$ переменных разложим по $n-2$ первым переменным и реализуем все конъюнкции длины $n-2$ полным дихотомическим деревом. Теперь, используя легко проверяемое равенство $L_{\pi}^{0}(2)=5$, получаем соотношение

$$
L_{\pi}^{0}(n) \leqslant \frac{7}{4} 2^{n}
$$

В предлагаемом ниже методе синтеза $\pi$-схем, дающем более точную, чем в (5), оценку константы $\sigma$, используется предложенное О.Б. Лупановым разложение булевых функций [5].

Всюду в дальнейшем через $\log n$ будем обозначать $\log _{2} n$. 
Лемма 1. Справедливо неравенство

$$
L_{\pi}^{0}(n) \leqslant 2^{n}\left(1+\frac{\log n}{n}+o\left(\frac{\log n}{n}\right)\right) .
$$

Доказательство. Пусть $f-$ произвольная булева функция от $n$ переменных. Введем параметр $r$ и переменные функции $f$ разобьем на две группы $x_{1}, x_{2}, \ldots, x_{r}$ и $y_{1}, y_{2}, \ldots, y_{n-r}$. Функцию $f$ будем задавать булевой $\left(2^{r} \times 2^{n-r}\right)$-матрицей $M^{f}$ следующим образом.

Через $\tilde{x}(\tilde{y})$ будем обозначать набор $\left(x_{1}, x_{2}, \ldots, x_{r}\right)$ (набор $\left(y_{1}, y_{2}, \ldots, y_{n-r}\right)$ ). Через $X_{j}\left(Y_{j}\right)$ будем обозначать набор $\tilde{x}$ (набор $\tilde{y}$ ), являющийся двоичной записью числа $j$. Нумерацию строк и столбцов матриц будем начинать с нуля. Элементом матрицы $M^{f}$, расположенным на пересечении $t$-й строки и $j$-го столбца, является значение $f\left(X_{t}, Y_{j}\right)$.

Введем параметр $s$ и матрицу $M^{f}$ разобьем на полосы, каждая из которых, кроме, быть может, последней, содержит $s$ строк. Число $p$ полос матрицы $M^{f}$ равно ] $2^{r} / s\left[\right.$. Число строк последней полосы матрицы $M^{f}$ будем обозначать через $s^{\prime}$. Через $A_{i}, 1 \leqslant i \leqslant p$, будем обозначать $i$-ю полосу матрицы $M^{f}$.

Пусть $f_{i}$ - функция, совпадающая с $f$ на полосе $A_{i}$ и равная 0 вне ее. Столбцы матрицы, соответствующей функции $f_{i}$, разбиваем на группы одинаковых между собой столбцов. Пусть $\tilde{\tau}-$ произвольный набор длины (точнее высоты) $s$ (или $s^{\prime}$, если $i=p$ ).

Обозначим через $B_{i, \tilde{\tau}}$ группу столбцов матрицы $M^{f}$, равных $\tilde{\tau}$ в полосе $A_{i}$ (для некоторых $(i, \tilde{\tau})$ группа $B_{i, \tilde{\tau}}$ может оказаться пустой). Пусть $f_{i, \tilde{\tau}}-$ функция, совпадающая с $f_{i}$ на столбцах из $B_{i, \tilde{\tau}}$ и равная 0 в остальных случаях (если $B_{i, \tilde{\tau}}$ - пустая группа, то соответствующая функция $f_{i, \tilde{\tau}}$ тождественно равна 0$)$.

Матрица для функции $f_{i, \tilde{\tau}}$ имеет столбцы 2-х сортов:

(a) столбцы из $B_{i, \tilde{\tau}}$, равные $\tilde{\tau}$ в полосе $A_{i}$ и состоящие из нулей вне $A_{i}$;

(b) остальные столбцы, состоящие сплошь из нулей.

Поэтому функция $f_{i, \tilde{\tau}}$ может быть представлена в виде конъюнкции двух функций, зависящих от $x_{1}, \ldots, x_{r}$ и $y_{1}, \ldots, y_{k}$ :

(1) функции $f_{i, \tilde{\tau}}^{(1)}(\tilde{x})$, в матрице которой все столбцы равны $\tilde{\tau}$ в полосе $A_{i}$ и состоят из нулей вне $A_{i}$;

(2) функции $f_{i, \tilde{\tau}}^{(2)}(\tilde{y})$, равной 1 на столбцах из $B_{i, \tilde{\tau}}$ и равной 0 в остальных случаях.

Таким образом, функцию $f$ можно представить в виде

$$
f(\tilde{x}, \tilde{y})=\bigvee_{i=1}^{p} \bigvee_{\tilde{\tau}} f_{i, \tilde{\tau}}^{(1)}(\tilde{x}) f_{i, \tilde{\tau}}^{(2)}(\tilde{y})
$$

Реализующую функцию $f \pi$-схему $S$ будем строить в соответствии с этим разложением. Она состоит из не более чем $p 2^{s}$ подсхем $S_{i, \tilde{\tau}}^{(2)}$, реализующих функции $f_{i, \tilde{\tau}}^{(2)}(\tilde{y})$ и из не более чем $p 2^{s}$ подсхем $S_{i, \tilde{\tau}}^{(1)}$, реализуюццих функции $f_{i, \tilde{\tau}}^{(1)}(\tilde{x})$.

Подсхемы $S_{i, \tilde{\tau}}^{(2)}$ строим в соответствии с СДНФ для функций $f_{i, \tilde{\tau}}^{(2)}(\tilde{y})$. 
Нетрудно проверить, что для любого $i$ наборы $\tilde{x}$, соответствующие строкам $i$-й полосы, имеют не более трех различных начал длины $r-[\log s]$. Отсюда и из (5) для любых $i$ и $n$ получаем оценку

$$
L_{\pi}\left(f_{i, \tilde{\tau}}^{(1)}(\tilde{x})\right) \leqslant 3(r-[\log s]+2 s) .
$$

Таким образом, имеют место следующие оценки

$$
\begin{aligned}
& \sum_{i=1}^{p} \sum_{\tilde{\tau}} L\left(S_{i, \tilde{\tau}}^{(2)}\right) \leqslant \frac{n-r}{s} 2^{n}\left(1+\frac{s}{2^{r}}\right), \\
& \sum_{i=1}^{p} \sum_{\tilde{\tau}} L\left(S_{i, \tilde{\tau}}^{(1)}\right) \leqslant 2^{s+r}\left(6+3 \frac{r}{s}\right)\left(1+\frac{s}{2^{r}}\right) .
\end{aligned}
$$

Выбирая $r=[\sqrt{n}]$ и $s=n-[\sqrt{n}]-[\log n]$, получаем завершающую доказательство леммы 1 оценку

$$
L(S) \leqslant 2^{n}\left(1+\frac{\log n}{n}+o\left(\frac{\log n}{n}\right)\right) .
$$

Теперь перейдем к получению более точной нижней оценки константы $\sigma$.

Пусть формула $\Phi$ задает однородный язык $\mathscr{L}$ в алфавите $E_{k}$. Скажем, что $(\pi, k)$ схема, соответствующая формуле $\Phi$, является однородной.

Дугу $(\pi, k)$-схемы, которой приписан символ $\alpha \in E_{k}$, назовем $\alpha$-дугой. Подсхему, состоящую из $k$ параллельно соединенных 0 -дуг, 1 -дуг,.., $(k-1)$-дуг, назовем $u$ дугой (универсальной дугой).

Вершины и дуги однородной $(\pi, k)$-схемы разобьем на ярусы следующим образом. Вход схемы отнесем к нулевому ярусу. Вершинами первого яруса считаем вершины, инцидентные входу. Вершинами $i$-го яруса, $i \geqslant 2$, считаем вершины, инцидентные вершинам $(i-1)$-го яруса и не являющиеся вершинами $(i-2)$-го яруса. Дуги, соединяющие вершины $(i-1)$-го и $i$-го ярусов, отнесем к $i$-му ярусу.

Отметим, что выход $(\pi, k)$-схемы, реализующей $n$-однородный язык, является вершиной $n$-го яруса.

Пусть $A$ и $B$ - вершины $i$-го и $j$-го яруса соответственно, $j>i$, произвольной однородной $(\pi, k)$-схемы $S$. Последовательность попарно инцидентных дуг $\left(p_{i}, p_{i+1}, \ldots, p_{j}\right)$, где $p_{s}$ - дуга $s$-го яруса, $p_{i}$ инцидентна вершине $A$ и $p_{j}$ инцидентна вершине $B$, назовем путем $\overline{A B}$.

Нетрудно проверить, что имеет место следующее утверждение.

Лемма 2. Произвольная $(\pi, k)$-схема, реализующая $n$-однородный язык, обладает следующими свойствами:

(1) любой путь, соединяющий вход и выход схемы, имеет длину $n$;

(2) все пути между любыми двумя вершинами имеют одинаковую длину;

(3) из любой вершины существует путь к выходу и из входа существует путь в любую вершину.

Лемма 3. . Пусть $A$ и $B$ - различнье вершины $i$-го яруса, и пусть $C$ u $D$ различные вершины $j$-го яруса, $j>i$, произвольной однородной $\pi$-сети $S$. Тогда если в сети $S$ существуют пути $\overline{A C}$ и $\overline{B C}$, то не существует пути $\overline{B D}$. 
Доказательство. Доказательство проведем рассуждением от противного. Пусть в сети $S$, удовлетворяющей условиям леммы, существует путь $\overline{B D}$. По свойству 3 однородных $(\pi, k)$-схем существуют пути из входа $a$ в вершины $A$ и $B$ и из вершин $C$ и $D$ к выходу $b$. Пусть $E$ и $F$ - общие точки этих путей из ярусов, имеющих наименьший и наибольший номер. Отметим, что вершина $E(F)$ может совпадать с входом $a$ (с выходом $b)$. Подсеть с полюсами $E$ и $F$, содержащая пути $\overline{E A}, \overline{E B}, \overline{A C}$, $\overline{B C}, \overline{B D}, \overline{C F}$ и $\overline{D F}$ является $H$-сетью. Следовательно, сеть $S$ не является $\pi$-сетью. Полученное противоречие завершает доказательство леммы.

Следствием леммы 3 является следующее утверждение.

Лемма 4. Пусть $A$ и $B$ - различные вершины $i$-го яруса, и пустъ $C$ u $D$ - различные вериины $j$-го яруса, $j>i$, произвольной однородной $\pi$-сети $S$. Тогда если в сети $S$ существуют не имеющие общих вершин пути $\overline{A C}$ и $\overline{B D}$, то не существуem nymu $\overline{B C}$.

Пусть $S$ - произвольная однородная $\pi$-сеть, не содержащая кратных дуг. Из лемм 3 и 4 следует, что если полустепень исхода (захода) вершины сети $S$ больше 1 , то полустепень захода (исхода) смежных с ней вершин следующего (предыдущего) яруса равна 1 . Сети $S$ поставим в соответствие код в алфавите $\{b, d, z\}$ следующим образом. Дуги сети будем обходить слева направо в пределах яруса в порядке увеличения номеров ярусов. Кодом сети $S$ является конкатенация кодов ее дуг (или групп ее дуг).

Группу всех дуг, инцидентных вершине с полустепенью исхода (захода) $r>1$, кодируем набором $b^{r-1} z$ (набором $d^{r-1} z$ ). Каждую оставшуюся дугу кодируем символом $z$.

Заметим, что сеть $S$ однозначно восстанавливается по своему коду.

Пусть $N_{1}(l)$ - множество попарно неизоморфных однородных $\pi$-сетей, имеющих $l$ дуг и не содержащих кратных дуг. Из вышеизложенного следует, что $\left|N_{1}(l)\right|$ не превосходит числа наборов длины $l$ в алфавите $\{b, d, z\}$, в которых после символа $b$ (символа $d$ ) не может следовать символ $d$ (символ $b$ ).

Оценим мощность множества $M(l)$ всех таких наборов. Через $M(l, q, m)$ обозначим множество наборов из $M(l)$, в которых число поднаборов $b^{i-1} z$ или $d^{i-1} z, i \geqslant 2$, равно $q$, а общее число символов $b$ и $d$ равно $m+q$. Отметим, что $0 \leqslant m \leqslant l$, $q \leqslant(l-m) / 2$.

Разобьем $m+q$ символов на $q$ непустых групп, каждая из которых состоит из одинаковых букв, $b$ или $d$. Символы $z$ вне поднаборов, их $(l-m-2 q)$ штук, разобьем на $q+1$ групп (группы могут быть и пустыми).

Таким образом,

$$
|M(l, q, m)| \leqslant 2^{q}\left(\begin{array}{c}
m+q-1 \\
q-1
\end{array}\right)\left(\begin{array}{c}
l-m-q \\
q
\end{array}\right) .
$$

Нетрудно проверить, что для любых $q$ и $m$

$$
|M(l, q, m)| \leqslant 2^{l+q-1}
$$

и

$$
\log |M(l,(1 / 4) l,(1 / 4) l)| \sim(5 / 4) l .
$$


Следовательно, для оценки максимального значения $|M(l, q, m)|$ мы будем рассматривать случай $q \geqslant(1 / 4) l$. При фиксированном $q$ значение $m$, при котором $|M(l, q, m)|$ максимально, равно

$$
\frac{(q-1) l}{2 q-1}-q
$$

Максимум произведения

$$
\left(\begin{array}{c}
l / 2 \\
q
\end{array}\right)\left(\begin{array}{c}
l / 2 \\
q
\end{array}\right) 2^{q}
$$

достигается при $q \sim l /(2+\sqrt{2})$.

Таким образом, получаем оценку

$$
\log \left(\max _{q, m}|M(l, q, m)|\right) \lesssim 1,2715 l,
$$

из которой следует оценка

$$
\log \left|N_{1}(l)\right| \lesssim 1,2715 l
$$

Теперь оценим мощность множества $N(l)$ неизоморфных $\pi$-схем, имеющих $l$ дуг. Пусть $N(l, r)$ - множество $\pi$-схем из $N(l)$, имеющих $r, r \leqslant l / 2, u$-дуг.

Выбираем дуги, входящие в состав $u$-дуг. Это можно сделать $\left(\begin{array}{c}l-r \\ r\end{array}\right)$ способами. Оставшиеся $l-2 r$ дуг занумеруем символами 0 или 1 . Отсюда и из (6) получаем оценку

$$
|N(l, r)| \leqslant\left(\begin{array}{c}
l-r \\
r
\end{array}\right) 2^{l-2 r} 2^{1,2715(l-r)} .
$$

Отсюда, используя свойства функции $\left(\begin{array}{l}n \\ k\end{array}\right)$, получаем оценку

$$
\log |N(l)| \lesssim 2,4015 l .
$$

Лемма 5. Справедливо соотночение

$$
L_{\pi}^{0}(n) \gtrsim 0,4164 \cdot 2^{n}
$$

Доказательство следует из (7) и обычных мощностных рассуждений. Из лемм 1 и 5 и (1) получаем следующее утверждение.

Теорема 2. При $n \rightarrow \infty$

$$
L_{\Phi}^{0}(n) \sim \sigma 2^{n}
$$

где $\sigma$ - константа из интервала $[0,4164,1]$.

\section{3. Сложность реализации дизъюнкций}

Нетрудно проверить, что все функции из $D_{n}$ имеют одинаковую сложность реализации. Поэтому будем рассматривать реализацию чистой дизъюнкции $x_{1} \vee x_{2} \vee \ldots \vee x_{n}$.

Лемма 6. Справедливо соотночение

$$
L_{\pi}\left(D_{n}\right) \lesssim \frac{3}{2} n \log n .
$$


Доказательство. Пусть $S-\pi$-схема, являющаяся $p$-схемой и состоящая из двух компонент $S_{1}$ и $S_{2}$. Подсхема $S_{1}\left(S_{2}\right)$ является последовательным соединением подсхем $S_{1}^{1}$ и $S_{1}^{2}\left(S_{2}^{1}\right.$ и $\left.S_{2}^{2}\right)$. Подсхема $S_{1}^{1}\left(S_{2}^{2}\right)$ реализует чистую дизъюнкцию от $n-[n / 2]$ $([n / 2])$ переменных. Подсхема $S_{1}^{2}\left(S_{2}^{1}\right)$ является последовательным соединением $[n / 2]$ $(n-[n / 2]) u$-дуг (0-дуг).

Нетрудно проверить, что схема $S$ реализует дизъюнкцию $x_{1} \vee x_{2} \vee \ldots \vee x_{n}$ и имеет место оценка

$$
L_{\pi}\left(D_{n}\right) \leqslant L_{\pi}\left(D_{[n / 2]}\right)+L_{\pi}\left(D_{n-[n / 2]}\right)+2[n / 2]+n-[n / 2] .
$$

Реализуя аналогичным образом схемы $S_{1}^{1}$ и $S_{2}^{2}$ и используя очевидную оценку $L_{\pi}\left(D_{1}\right)=1$, получаем утверждение леммы 6 .

Для получения нижних оценок функционала $L_{\pi}\left(D_{n}\right)$ будем использовать следующую легко проверяемую лемму.

Лемма 7. Функционирование $\pi$-схемы, реализующей чистую дизғюнкцию, не изменится, если в ней любые 0-дуги заменить и-дугами.

Состоящую из 1 -дуг и $u$-дуг $\pi$-схему назовем $(\pi, 1, u)$-схемой. При этом $u$-дугу считаем одним элементом. Сложностью $(\pi, 1, u)$-схемы будем называть число ее 1 -дуг и $u$-дуг. Через $L^{\prime}(d, n)$ обозначим минимальную сложность $(\pi, 1, u)$-схемы, peализующей чистую дизъюнкцию от $n$ переменных.

Нетрудно проверить, что

$$
L_{\pi}\left(D_{n}\right) \geqslant L^{\prime}(d, n)
$$

Содержащую путь из $u$-дуг, соединяющий ее вход и выход, $\pi$-схему будем называть $u$-схемой; $(\pi, 1, u)$-схему, не являющуюся $u$-схемой, назовем $\bar{u}$-схемой; $\pi$-схему, состоящую из последовательно соединенных $u$-дуг, назовем $u$-цепью.

Нетрудно проверить, что имеет место следующее утверждение.

Лемма 8. В минималъной $(\pi, 1, u)$-схеме, реализующей чистую дизғюнкцию, любая являющаяся и-схемой подсхема есть и-цепь, любая являющаяся р-схемой подсхема есть и-схема и любая являющаяся $s$-схемой подсхема содержит не более одной компоненты, являющейся $р$-схемой.

Пусть $U$ - множество $(\pi, 1, u)$-схем. Пусть $R_{1}\left(R_{2}\right)$ - множество $s$-схем из $U$, первая (последняя) компонента которых является $\bar{u}$-схемой, а остальные компоненты $u$-дуги. Пусть $R_{3}$ - множество не являющихся $u$-схемами $s$-схем из $U$, и первая и последняя компонента которых является $u$-дугой. Пусть $R=R_{1} \cup R_{2} \cup R_{3}$.

Лемма 9. Минимальная $(\pi, 1, u)$-схема, реализующая чистую дизғюнкцию от $n \geqslant 2$ переменных, является $p$-схемой из двух компонент, одна из которых яв-

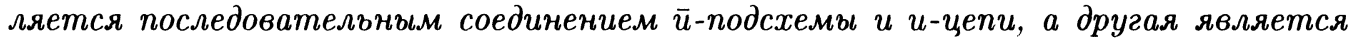

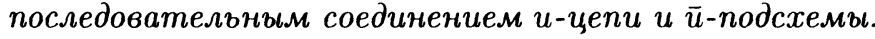

Доказательство. Пусть $S$ - произвольная $(\pi, 1, u)$-схема, удовлетворяющая условиям леммы. Используя лемму 8 , нетрудно проверить, что схема $S$ является $p$-схемой, компоненты которой принадлежат множеству $R$.

Нетрудно проверить, что $\pi$-схема, в которой имеется вершина с большей единицы полустепенью захода (исхода) такая, что всем входящим в (исходящим из) нее 
дугам приписан один и тот же символ, не является минимальной. Таким образом, имеющая две компоненты из множества $R_{1}\left(R_{2}, R_{3}\right)$ p-схема не минимальна. Имеющая компоненту из множества $R_{1}\left(R_{2}\right)$ и компоненту из множества $R_{3} p$-схема, также, не минимальна.

Лемма 9 доказана.

Лемма 10. В минимальной $(\pi, 1, u)$-схеме, реализующей чистую дизғюнкцию от $n \geqslant 2$ переменных, сумма глубин $\bar{u}$-подсхем ее двух компонент равна $n$.

Доказательство. Доказательство проведем рассуждением от противного. Пусть $S$ минимальная $(\pi, 1, u)$-схема, реализующая чистую дизъюнкцию от $n \geqslant 2$ переменных, состоящая (согласно лемме 9) из двух компонент $S_{1}$ и $S_{2}$. Подсхема $S_{1}\left(S_{2}\right)$ является последовательным соединением подсхем $S_{1}^{1}$ и $S_{1}^{2}\left(S_{2}^{1}\right.$ и $\left.S_{2}^{2}\right)$. Пусть подсхема $S_{1}^{1}\left(S_{2}^{2}\right)$ является $\bar{u}$-подсхемой и имеет глубину $m_{1}\left(m_{2}\right)$. Подсхема $S_{1}^{2}\left(S_{2}^{1}\right)$ является последовательным соединением $n-m_{1}\left(n-m_{2}\right) u$-дуг.

Пусть $m_{1}+m_{2}<n$. Подсхема $S_{1}^{1}\left(S_{2}^{2}\right)$ не проводит на наборе $0^{m_{1}}\left(0^{m_{2}}\right)$. Схема $S$ не проводит на $2^{n-m_{1}-m_{2}}$ наборах $\left(0^{m_{1}} \tilde{\gamma} 0^{m_{2}}\right)$, где $\tilde{\gamma}-$ произвольный булев набор длины $n-m_{1}-m_{2}$. Получаем противоречие, так как по определению схема $S$ не проводит на единственном наборе $0^{n}$.

Таким образом, $m_{1}+m_{2} \geqslant n$.

Пусть теперь $m_{1}+m_{2}>n$ и пусть в одной из $\bar{u}$-подсхем, например, в $S_{1}^{1}$ нет $u$-пути длины $n-m_{2}$, инцидентного входу схемы $S$. В этом случае приписывание всем дугам подсхемы $S_{1}^{1}$ из ярусов с $\left(n-m_{2}\right)$-го по $m_{1}$-й символа $u$ не изменит функционирования схемы $S$ (не появится путь, соответствующий набору $0^{n}$ ). Следовательно, подсхема $S_{1}^{1}$ не минимальна (в ней всем вершинам, инцидентным выходу, стал приписан символ $u$ ).

Пусть, наконец, $m_{1}+m_{2}=n$ и пусть в подсхеме $S_{1}^{1}\left(S_{2}^{2}\right)$ есть инцидентный входу (выходу) $u$-путь $W_{1}\left(W_{2}\right)$ длины $n-m_{2}\left(n-m_{1}\right)$.

Через $A(D)$ обозначим конечную (начальную) вершину пути $W_{1}\left(W_{2}\right)$, а через $B$ $(C)$ - выход (вход) подсхемы $S_{1}^{1}\left(S_{2}^{2}\right)$. Нетрудно проверить, что если из подсхемы $S_{1}^{1}$ удалить ее подсхему с входом $A$ и выходом $B$ и перенести последнюю в подсхему $S_{2}^{2}$, объединив вершины $A$ и $C$ и вершины $B$ и $D$, то функционирование не изменится. Можно проверить, что новая схема не минимальна. Следовательно, не минимальна и исходная схема.

Лемма 10 доказана полностью.

\section{Лемма 11. Справедливо соотношение}

$$
L_{\pi}\left(D_{n}\right) \gtrsim n \log n
$$

Доказательство. Доказательство проведем по индукции. В качестве базы индукции возьмем случай $n=1$, в котором $L_{\pi}\left(D_{1}\right)=L^{\prime}(d, 1)=1 \geqslant 1 \cdot \log 1$.

Пусть $S$ - минимальная $(\pi, 1, u)$-схема, реализующая чистую дизъюнкцию от $n \geqslant 2$ переменных, состоящая (согласно лемме 9) из двух компонент $S_{1}$ и $S_{2}$. Подсхема $S_{1}\left(S_{2}\right)$ является последовательным соединением подсхем $S_{1}^{1}$ и $S_{1}^{2}\left(S_{2}^{1}\right.$ и $\left.S_{2}^{2}\right)$. Пусть подсхема $S_{1}^{1}$ является $\bar{u}$-подсхемой и имеет глубину $m, 1 \leqslant m \leqslant n-1$. Тогда согласно лемме 10 являющаяся $\bar{u}$-подсхемой подсхема $S_{2}^{2}$ имеет глубину $n-m$.

Нетрудно проверить, что подсхема $S_{1}^{1}\left(S_{2}^{2}\right)$ реализует чистую дизъюнкцию от $m$ $(n-m)$ переменных. 
Таким образом, получена точная оценка

$$
L^{\prime}(d, n)=n+\min _{m}\left(L^{\prime}(d, m)+L^{\prime}(d, n-m)\right) .
$$

Нетрудно проверить, что

$$
\min _{m}(m \log m+(n-m) \log (n-m))
$$

достигается при $m=[n / 2]$. Отсюда, из (8) и (9) следует справедливость индуктивного перехода.

Лемма 11 доказана.

Из лемм 6 и 11 и (1) получаем следующее утверждение.

Теорема 3. Справедливы соотношения

$$
n \log n \lesssim L_{\Phi}\left(D_{n}\right) \lesssim \frac{3}{2} n \log n
$$

\section{4. Функции, обращающиеся в единицу на $r$ наборах}

Лемма 12. Для любых $n u r, r \leqslant 2^{n}$,

$$
L_{\pi}\left(F_{n, r}\right) \leqslant(n-] \log r[+1) r+2^{] \log r[}-2 .
$$

Доказателъство. Пусть $f-$ произвольная функция из $F_{n, r}$. Обозначим через $M_{f}$ множество всех начал длины $] \log r\left[\right.$ наборов из $N_{f}$. Отметим, что $\left|M_{f}\right| \leqslant r$. Строим $\pi$-схему $S$, реализующую функцию $f$, которая содержит $\left|M_{f}\right|$ вершин $] \log r$ [-го яруса. Пусть $S_{1}\left(S_{2}, S_{3}\right)$ - часть схемы $S$, содержащая дуги ярусов с 1-го по (] $\log r[-1)$-й (] $\log r\left[-\right.$-го, с (] $\log r[+1)$-го по $n$-й). Отметим, что часть $S_{3}$ может быть пустой.

Ясно, что $L\left(S_{1}\right) \leqslant 2 \cdot 2^{\log r[-1}-2$, так как подсхема $S_{1}$ не более сложна, чем полное дихотомическое дерево глубины $] \log r[-1$.

Нетрудно проверить, что имеют место оценки

$$
L\left(S_{2}\right)=\left|M_{f}\right| \leqslant r, \quad L\left(S_{3}\right) \leqslant(n-] \log r[) r .
$$

Лемма доказана.

Через $a^{m}$ будем обозначать набор длины $m$, все компоненты которого равны $a$.

Пусть $G_{n, r, p}$ - множество булевых функций от $n$ переменных, обращающихся в единицу на $r$ произвольных различных наборах вида

$$
\left(\sigma_{1}, \ldots, \sigma_{p}, 0^{n-2 p}, \sigma_{1}, \ldots, \sigma_{p}\right),
$$

где $r \leqslant 2^{[n / 2]}$ и $] \log r[\leqslant p \leqslant[n / 2]$.

Нетрудно проверить, что произвольная минимальная $\pi$-схема $S$, реализующая функцию из $G_{n, r, p}$, обладает следующими свойствами:

(1) схема $S$ не содержит $u$-дуг;

(2) ее подсхемы $S_{1}$ и $S_{3}$, содержащие дуги ярусов с 1-го по $p$-й и с $(n-p+1)$-го по $n$-й, соответственно, не содержат $p$-подсхем; 
(3) полустепень захода любой вершины подсхемы $S_{1}$ равна 1 ;

(4) полустепень исхода любой вершины подсхемы $S_{3}$ равна 1 ;

(5) полустепени захода и исхода любой вершины подсхемы $S_{2}$, содержащей дуги ярусов с $(p+1)$-го по $(n-p)$-й, совпадают и равны 1 .

Пусть $g_{n, l, p}$ - произвольная функция из $G_{n, l, p}, l \geqslant 2$ и пусть $g_{n, l-1, p}^{\prime}-$ функция из $G_{n, l-1, p}$, отличающаяся от $g_{n, l, p}$ на одном произвольном наборе.

Лемма 13. Справедливо неравенство

$$
L_{\pi}\left(g_{n, l, p}\right) \geqslant L_{\pi}\left(g_{n, l-1, p}^{\prime}\right)+n-p+1 .
$$

Доказательство. Пусть $S$ - минимальная $\pi$-схема, реализующая функцию $g_{n, l, p}$. Пусть

$$
\tilde{\alpha}=\left(\alpha_{1}, \ldots, \alpha_{p}, 0^{n-2 p}, \alpha_{1}, \ldots, \alpha_{p}\right)
$$

- набор, различающий функции $g_{n, l, p}$ и $g_{n, l-1, p}^{\prime}$, и пусть $A(B)$ - имеющая степень, большую 2 , вершина в подсхеме $S_{1}\left(S_{3}\right)$ на соответствующем этому набору пути $W$, принадлежащая ярусу с наибольшим (наименьшим) номером $i(n-p+j)$.

Удаляя из схемы $S$ путь

$$
W_{1}=\left(\alpha_{i+1}, \ldots, \alpha_{p}, 0^{n-2 p}, \alpha_{1}, \ldots, \alpha_{j}\right),
$$

являющийся частью пути $W$, получим схему $S^{\prime}$, реализующую функцию $g_{n, l-1, r}^{\prime}$.

Нетрудно проверить, что

$$
L_{\pi}\left(g_{n, l-1, p}^{\prime}\right) \leqslant L\left(S^{\prime}\right)=L_{\pi}\left(g_{n, l, p}\right)-(n-p-i+j) .
$$

Покажем, что $n-p-i+j \geqslant n-p+1$, то есть что $j>i$. Существует отличный от пути $W_{1}$ путь $W_{2}$ из вершины $A$ в вершину $B$, так как в противном случае существуют отличная от вершины $A$ вершина $C i$-го яруса и отличная от вершины $B$ вершина $D(n-p+j)$-го яруса такие, что пути $\overline{A D}$ и $\overline{B C}$ не пересекаются, что противоречит лемме 3 .

Из минимальности схемы $S$ следует, что начальные (конечные) дуги путей $W_{1}$ и $W_{2}$ имеют противоположную нумерацию.

Из определения функции $g_{n, l, p}$ следует, что части путей $W_{1}$ и $W_{2}$, лежащие в ярусах с номерами с $(n-p+1)$-го по $(n-p+i)$-й, одинаковы. Следовательно, $j \geqslant i+1$. Лемма доказана.

Лемма 14. Справедливо неравенство

$$
L_{\pi}\left(G_{n, r, p}\right) \geqslant r(n-p+1) .
$$

Доказательство получаем последовательным применением леммы 13 к произвольной функции из $G_{n, r, p}$.

Теорема 4. Для любого $r \leqslant 2^{[n / 2]}$

$$
L_{\Phi}\left(F_{n, r}\right) \sim r(n-\log r) .
$$

Доказателъство. Верхняя оценка следует из леммы 12. Полагая $p=] \log r[$ в лемме 14, получаем нижнюю оценку. 
Замечание 1. Функции из множества $G_{n, r, p}$ являются примерами индивидуальных функций, имеющих при $r \asymp n$ зкспоненциальную сложность реализации.

Теорема 5. При $r=2^{c n}, c \in[1 / 2,1)$,

$$
L_{\Phi}\left(F_{n, r}\right) \asymp r(n-\log r) .
$$

Доказательство. В условиях теоремы

$$
\left|F_{n, r}\right|=\left(\begin{array}{c}
2^{n} \\
2^{c n}
\end{array}\right) \sim \frac{2^{n 2^{c n}}}{\left(2^{c n}\right) !} .
$$

Отсюда, $\log \left|F_{n, r}\right| \sim(1-c) n 2^{c n}$. Из (7) и обычных мощностных рассуждений получаем нижнюю оценку.

Разлагая функцию $f$ по первым ] $c n$ [ переменным и реализуя функции от оставшихся переменных схемами в классе СДНФ, получаем верхнюю оценку.

Теорема 5 доказана.

Через $B_{\Phi}(M)$ обозначим минимум $L_{\Phi}(\mathscr{L})$ по всем языкам $\mathscr{L}$ из множества $M$. Нетрудно проверить, что имеет место следующее утверждение.

Теорема 6. Для любого $r$

$$
B_{\Phi}\left(F_{n, r}\right) \lesssim n-\log r+\log r \log \log r .
$$

\section{5. Основные результаты для произвольных конечных языков}

Вначале рассмотрим случай произвольных конечных булевых языков. Нетрудно проверить, что

$$
L_{\pi}(n) \leqslant \sum_{i=1}^{n} L_{\pi}^{0}(i) .
$$

Отсюда и из теоремы 2 получаем следующее утверждение.

Теорема 7. $\Pi p u n \rightarrow \infty$

$$
L_{\Phi}(2, n) \sim \sigma_{1} 2^{n}
$$

где $\sigma_{1}$ - константа из интервала $[0,4164,2]$.

Теперь обобщим полученные выше результаты на случай конечных языков регулярных выражений в алфавите $E_{k}, k \geqslant 3$.

Из оценки числа $\pi$-сетей [7] и аналогов теоремы 2 и соотношения (10) для случая произвольных $k$ получаем следующие утверждения.

Теорема 8. Для любого $k \geqslant 3$ nри $n \rightarrow \infty$

$$
L_{\Phi}^{0}(k, n) \sim \sigma_{2} k^{n}
$$

где $\sigma_{2}$ - зависящая от $k$ константа такая, что

$$
\frac{1}{4+\log k} \leqslant \sigma_{2} \leqslant \frac{1}{\log k} .
$$


Теорема 9. Для любого $k \geqslant 3$ nрu $n \rightarrow \infty$

$$
L_{\Phi}(k, n) \sim \sigma_{3} k^{n}
$$

где $\sigma_{3}$ - зависящая от $k$ константа такая, что

$$
\frac{1}{4+\log k} \leqslant \sigma_{3} \leqslant \frac{1}{\log k} \frac{k}{k-1} .
$$

Отметим, что нижнюю оценку теоремы 8 можно улучшить, применяя рассуждения, аналогичные приведенным в параграфе 2 .

Аналоги результатов, полученных в параграфах 3 и 4 , имеют место и для случая произвольного $k \geqslant 3$.

В заключение, выражаю глубокую благодарность О.Б. Лупанову за внимание к работе.

\section{Список литературы}

1. Глушков В.М., Синтез цифровых автоматов. Физматгиз, Москва, 1962.

2. Клини С.К., Представление событий в нервных сетях и конечных автоматах. Автоматы. ИЛ, Москва, 1956, 15-67.

3. Лупанов О.Б., О реализации функций алгебры логики формулами из конечных классов (формулами ограниченной глубины) в базисе $\&, \vee, \neg$. Проблемъ кибернетики (1961) 6, 5-14.

4. Лупанов О.Б., О синтезе некоторых классов управляющих систем. Проблемъ кибернетики (1963) 10, 88-96.

5. Орлова Е.В., Реализация булевых функций регулярными выражениями. Тезисы докладов 11-й международной конференции по проблемам теоретической кибернетики. РГГУ, Москва, 1996.

6. Орлова Е.В., О сложности регулярных выражений для функций из некоторых классов. Тезисъ докладов 12-й международной конференции по проблемам теоретической кибернетики. Изд-во мех.-мат. ф-та МГУ, Москва, 1999.

7. Яблонский С.В., Введение в дискретную математику. Наука, Москва, 1979.

Статья поступила 17.01.2000. 